\title{
XI.
}

\section{Ein Rhabdomyosarcom mit hyaliner Degeneration (Cylindrom) im willkürlichen Muskel.}

(Beiträge zur pathologischen Anatomie des quergestreiften

Muskels:)

(Aus dem pathologischen Institut in Strassburg i. E.)

Von

Dr. med. Akira Fajinami aus Japan.

(Hierzu Tafel XII, Fig. 3 und 4 im Bd. 159, Heft 2.')

Wie das sogenannte Cylindrom Billroth's schon als ein seltenes Vorkommniss unser Interesse in Anspruch nimmt, ebenso selten und wichtig erscheint diejenige Geschwulst, welche durch das Vorkommen quergestreifter Zellen und Fasern ausgezeichnet ist, die den embryonalen Muskelfasern gleichen. Diese Rhabdow yome wurden seit Rokitansky (in der Albuginea des Hodens ${ }^{2}$ )) wiederholt beobachtet und zwar besonders an Urogenital-Organen, am häufigsten in der Niere und Nebenniere, dann im Hoden, Uterus, Vagina, Ovarium und in der Harnblase. Von anderen Organen sind dagegen nur vereinzelte Fälle berichtet: in der Parotis, Nase, Orbita, Halsgegend, am Ausgang des Beckens, in dem Retroperitonalen Gewebe, Mediastinum in der Mamma und in anderen verschiedenen Körpertheilen, wie Sacral-, Unterkiefer-, Schläfengegend. Solche Geschwülste mit quergestreiften Muskelfasern kommen fast ausnahmslos entweder bei Neugeborenen, oder bei jugendlichen Individuen zur Beobachtung. Die quergestreiften

1) Diese Abbildungen sind irrthümlicherweise in dem betreffenden Heft auf einer mit No. XI bezeichneten Tafel veröffentlicht worden.

2) Rokitansky, (Ein aus quergestreiften Muskelfasern constituirtes Aftergewebe.) Zeitschrift der Gesellschaft der Wiener. Aerzte 1849. S. 331 . 

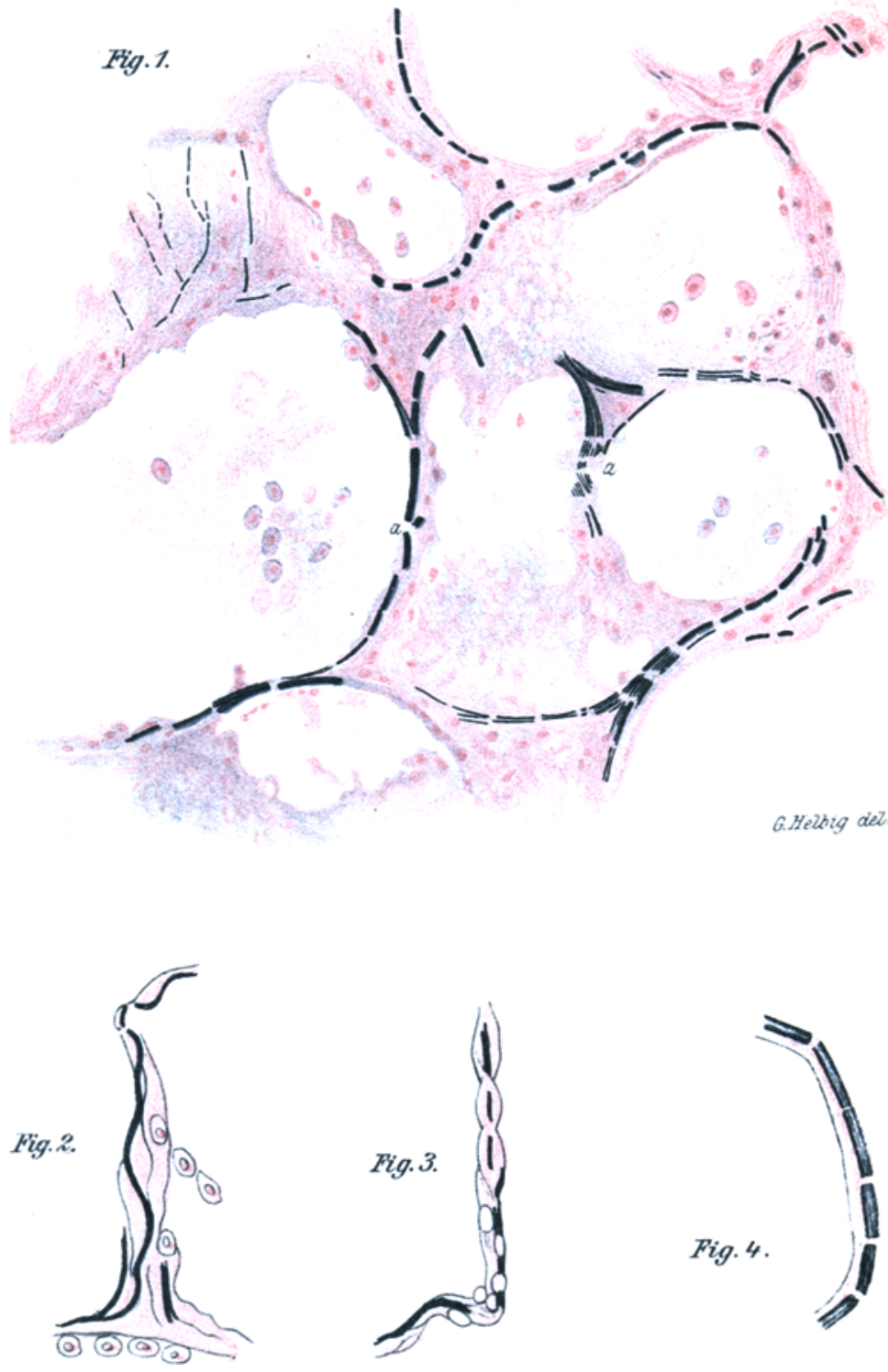
Muskelfasern stellen entweder die Haupt-Elemente der Geschwulst dar, oder sie erscheinen nur in spärlicher Menge, wäbrend andere Geschwulstzellen vorherrschen.

Die meisten Autoren sind geneigt, diese Elemente auf versprengte embryonale Muskelkeime zurückzufübren, sobald die Organe, in denen die Geschwülste gewachsen sind, gar kein Muskelgewebe enthalten, von welchen diese specifischen Geschwulstzellen ausgegangen sein könnten. Auch für den Fall, dass die Geschwülste in den Organen mit glatten Muskelfasern entwickelt sind, z. B. im Uterus oder Oesophagus ${ }^{1}$ ), herrscht die Meinung vor, dass ihr Ursprung ein embryonaler sei. So hat Ribbert seine frühere Ansicht ${ }^{2}$ ), dass eine Umwandlung von glatten Fasern in quergestreifte stattfinde, aufgegeben und behauptet jetzt ihre Herkunft aus embryonalen Keimen ${ }^{3}$ ). Auch einem Rhabdomyome des Speiserohrs bei einem 75jährigen Manne hat $W$ olfensberger ${ }^{4}$ ) einen embryonalen Ursprung zagesprochen; trotz des Nachweises reichlicher Spindelzellen will er absichtlich den Namen "Sarcom" vermeiden, geht vielmehr von der Annahme aus, dass diese Spindelzellen alle ein Vorstadium der quergestreiften Muskelzellen darstellen.

In Organen mit quergestreiften Muskelfasern, und zwar im Herzen, hat zuerst von Recklinghausen ${ }^{5}$ ) bei einem Neugeborenen mehrere bis taubenei-grosse "Myome" gesehen, denen späterhin von Zenker ${ }^{6}$ ) der Name "Rhabdomyom" gegeben wurde.

1) Hanau: Gesellschaft d. Aerzte in Zürich'. Sitzung vom 24. Juli 1892. Wolfensberger, über ein Rbabdomyom der Speiseröbre. Zi egler's Beiträge. Bd. 15. S. 49). 1894.

$\left.{ }^{2}\right)$ Ribbert: Ueber ein Myosarcoma striocellulare des Nierenbeckens und des Ureters. Dieses Archiv CVI. S. 282.

3) Ribbert: Beiträge zur Kenntniss der Rhabdomyome. Dieses Archiv CXXX. S. 249.

4) Wolfensberger a. a. 0 .

5) v. Recklinghausen: Verhandlung der Gesellschaft für Geburtshülfe in Berlin; Sitzung vom 25. März 1862. Monatsschrift für Geburtskunde XX, S. 1, 1862.

6) Zenker: Ueber die Veränderungen der willkürl. Muskeln im Typhus abdom. Leipzig 1864: S. 85. 
Aehnliche Fälle wurden seitdem noch von anderen Autoren beschrieben. In derZunge fand C. 0 . W eber ${ }^{1}$ ) ein wabres Myom, aber in der Körper-Musculatur ist bis jetzt eine Muskelgeschwulst mit quergestreifter Faser fast gar nicht bekannt. 1856 hat Billroth ${ }^{2}$ ) einen 'Tumor „Myoma cysticum“ am linken Oberarm beschrieben, welcher vom Muskel selbst ausgegangen sein soll. Durch eigenthümliche, mit Fortsätzen versehene Zellen, welche wegen ihres grossen Kerngehalts einerseits mit den "Plaques à plusieurs nojeaux" Robin's verglichen werden konnten, andererseits den Entwickelungs-Formen der quergestreiften Muskelfasern (von. B. in einer andern Arbeit ${ }^{3}$ ) geschildert) sehr ähnlich erschienen, wurde Billroth veranlasst, auch diesen Tumor als eine richtige Muskelgeschwulst aufzufassen, wenn auch eine deutliche Querstreifung fehlte. Dass aber dieser Fall zweifelhaft ist, hat $B$. sogleich hervorgehoben und auch späterhin nochmals ausgesprochen ${ }^{4}$ ) Die zweite Arbeit über den Muskeltumor mit quergestreiften Fasern wurde 1860 von $\mathrm{Lambl}^{5}$ ) publicirt, ein "Muskel-Carcinom", welches sich bei einem 3jährigen Mädchen augenscheinlich von der Tibia aus entwickelt hatte. In dieser Geschwulst (nicht Carcinom im heutigen, histogenetischen Sinne) hat L. neben den Haupt-Elementen nehmlich den spindelförmigen Zellen, zahlreiche bandartige Zellen wahrgenommen, welche er, da sie deutlich quer-, zum Theil auch Iängsgestreift erschienen, als unzweifelhafte, neugebildete längsgestreifte Muskelfasern betrachten durfte. Hiergegen wurde bald der. Einwand erhoben, dass es sich nur um übrig gebliebene Fasern des Wadenmuskels gehandelt

1) C. O. Weber, Anatomische Untersuchung einer hypertrophischen Zunge nebst Bemerkungen über die Neubildung quergestreifter Muskelfasern. Dieses Archiv Bd. VII. S. 115. 1854.

2) Billroth, über eine eigenthümlicbe Geschwulst der Muskeln. Dieses Archiv Bd. IX. S. 172. 1856.

3) Billroth, Zur Entwicklungsgeschicbte und chirurg. Bedeutung des Boden-Cystoids. Dieses Archiv Bd. VIII. S. 268. 1855.

4) Billroth, Beiträge zur patholog. Histologie. S. 61. (Geschwälste im Muskel).

5) Lambl, Carcinoma tibiae mit Neubildung von quergestreiften Muskelfasern. Aus d. Franz-Josef-Kinderhospitale in Prag. Theil I. S. 191. 1860. Canstatt's Jahresbericbt 1860 . II. S. 22. 
habe (Bardeleben ${ }^{1}$ ), Virchow ${ }^{2}$ ), während $Z$ enker ${ }^{3}$ ), wegen der offenbaren. Uebereinstimmung mit dem von Billroth im Hoden-Cystoid und von $Z$. in den regenerirenden Muskeln der Typhösen nachgewiesenen quergestreiften Elemente, an der Richitigkeit der Lambl'schen Deutung festgehalten hat. Die zwei Fälle von $\mathrm{Buhl}^{4}$, a) eine links neben der Lendenwirbelsäule gelegene Geschwulst bei einer 28jährigen Magd, b) eine dem

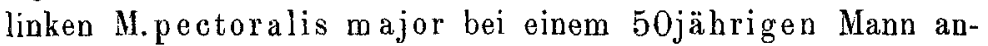
haftende, gehören zu den vom quergestreiften Muskelgewebe ausgegangenen Geschwülsten, weil sie aus quergestreiften Muskelfasern von verschiedener Entwickelungs-Stufe bestehen. Den Haupt-Accent legt Buhl darauf, dass eine Neubildung von Muskelzellen innerhalb des Muskels selbst stattgefunden habe. Seitdem findet sich in der Literatur kein derartiger Fall einer Geschwulst mit quergestreiften Muskelfasern, welche in der Körper-Musculatur gewachsen war. Es sind ja einige, quergestreifte Muskelfasern führende Tumoren in Gegenden beschrieben worden, in denen quergestreifte Muskeln vorkommen, so z. B. in der Sacralgegend, Unterkiefergegend, Schläfengegend u.s.w. Sie sind aber nicht hierher za rechnen, da ihre Bedeutung eine andere ist.

Die eben genannten Tumoren aus der Körper-Musculatur waren keine Mischgeschwülste, während in vielen Geschwülsten, die in nicht musculösen Organen auftreten, das rhabdomyomatöse Gewebe nur als einer der Bestandtheile neben dem Knorpelgewebe, Drüsengewebe u. s. w. erschien, das Ganze also als eine Mischgeschwulst bezeichnet werden musste, ein Verhalten, was bei manchen Autoren den Gedanken einer Entstehung aus verirrten embryonalen Keimen besonders förderte.

Das hiesige Pathologiscbe Institut besitzt seit vielen Jahren einen Tumor vom Unterschenkel eines 50 jährigen Mannes, welcher sich an ein chronisches, angeblich seit länger als 10 Jáhren bestehendes Haut-

1) Bardeleben, Chirurgie. I. Bd. 1863. S. 393. (Citirt von Zenker.)

2) Virchow, die krankhaften Geschwülste. III. S. 106.

3) Zenker, über die Veränderungen der willkärlichen Muskeln im Typhus abdominalis. Leipzig, 1864. S. 59.

4) Buhl, Wahres, revidirendes Myom. Zeitschrift für Biologie Bd. I. S. 263.1865 . 
gescbwür angeschlossen hatte und innerbalb 6 Wochen scbnell gewachsen war, weshalb die Amputatio femoris vorgenommen wurde. Der Tumor wurde sorgfältig in Spiritus aufbewabrt. Obwobl die Lage und Stelle der Geschwulst nicht mehr mit Sicherheit bestimmt werden kann, sehe jeh noch eine Thaler-grosse, scharf abgegrenzte Narbe an der Haut; dicht daneben ragt noch ein Hühnerei-grosser, böckeriger, leicht abgeplatteter Tumor nach aussen hervor, dessen Oberfläche leicht granulirt erscheint. Diesem angeschlossen, findet sich die Hauptmasse des Tumors, welche über Mannesfaust gross ist, weiter nach innen in der Musculatur und zu einem kleinen Theil dicht unter der Elaut mit dieser fest verwachsen. Sonst ist die Geschwulst nach allen Seiten von Muskelgewebe umgeben, welches mehr oder weniger stark atrophisch, aber stellenweise sehr schwer als solche zu erkennen ist. Leider konnte jch an diesem Präparate nicht wit Sicherheit constatiren, un welchen Unterschenkelmuskel es sich handelte. Die Consistenz ist durch die AlkoholConservirung ziemlich gross geworden, im Allgemeinen gleichmässig, nur der peripherische Theil fühlt sich weicher an, als der centrale. Der Uebergang zwischen beiden Abschnitten geschiebt ganz allmählich. Die neben der Hautnarbe hervorragende Partie fühlt sich ebenso derb an, ausserdem fällt an ihr partiell eine bräunlich-rotbe Farbe auf. An dem übrigen Präparat lässt sich, wohl weil es lange Zeit im Spiritus war, eine besondere Färbung nicht mebr bestimmen. So viel kann ich aber noch angeben, dass die centrale Partie der Geschwulst, welche sich derber als die peripherische anfühit, mehr weiss und mebr durchscheinend ist. Bei genauer Betrachtung sind Züge der einander durchflechtenden Bündel deutlich wahrzunehmen. Im Innern der Tumormasse ist kein anderwejtiger Bestandtbeil nachzuweisen, wie z. B. faseriges Muskelgewebe; Muskelfaserbündel sind erst an der peripherischen, höchstens $1 \mathrm{~cm}$ breiten Zone der Geschwulst sichtbar. In dieser Uebergangs-Zone ist die Grenze zwischen Geschwulst- und Muskelsubstanz ganz verwischt.

Dem Tumor, weleher mit der passendea Diagnose: "Cylindrom" aufbewahrt wurde, babe ich, da er je nach der Stelle ein recht wechselndes mikroskopisches Bild darbot, möglichst viele Präparate, und zwar aus den verscbiedensten Partien entnomimen. Besonders auffallend bei dem mikroskopischen Studium ist das reichliche Auftreten hyaliner Stränge zwischen den zelligen Elementen in Fast allen Abschnitten der Gesebwulst; besonders ausgedehnt sind sie im centralen Theil derselben, wo sie meist plexiform, bald gestreckt, bald gebogen, oder gar kolbig, entweder parallel nebeneinander aufgestellt oder verästelt erscheinen, und sogar mit einander communiciren können. Diese Stränge sind nicht immer gleich breit, im centralen Theil der Geschwulst sind sie immer viel dicker, als im peripherischen. Sie sind entweder ganz bomogen, oder nur ein bischen mit feinen, fibrillären Streifungen verseben, oder ganz leicht körnig. Mitten in wanchen feineren und dickeren hyalinen Sträugen ist ein feines, einfaches : Endothelrohr eingebettet, welches sich ohne besondere Unterlage direct an die hyaline Sub- 
stanz ausscbliesst und manchmal deutliche rothe Blutkörperchen enthält. Dieses Blutgefäss ist entweder eine richtige Capillare, in dem die Blutkörpercben nur eine einfache Reibe bilden, oder noch etwas grösser als ein Capillargefäss.

Díeses Verhalten der byalinen Stränge und ibre plexiforme Anordnung deuten wohl auf eine Entstehung der hyalinen Substanz durch die Degeneration der Blutgefässwand hin, wie sie von früheren Autoren gesehen wurde. (Billroth, Förster, von Recklingbausen, Kocber u. s.w.). Aber an anderen Stellen der Geschwulst ist dieses Verhalten ganz undeutlich, und ich konnte durch unmittelbares Zerzupfen, oder durch Maceration mit nachfolgendem Zerzupfen feine Netzwerke dieser byalinen Substanz isoliren: alsdann erscheiut der einzelne Strang sehr dünn, 5- und 10 inal dünner, als gewöhnliche Blutcapillaren. Meistentheils konnte ich aber Blutcapillaren inmitten dieser hyalinen Stränge nicht nachweisen. Hie und da, besonders im Centraltheil des Tumors, traf ich hyaline, rundliche Körper, welche entweder den Querschnitt der hyalinen Stränge oder den kolbig verdickten Theil derselben darstellen. Im Verhalten der byalinen Stränge, ist kein auffallender Unterscbied, mögen sie lầngsgeschnitten, d. i. parallel dem Verlauf der Muskelfaser, oder quergelagert sein: im ersteren Falle traf ich die längsverlaufenden hyalinen Stränge in der Mehrzahl. Der Rand des einzelnen Stranges ist meist glatt und scharf gegen die benachbarten Zellen abgegrenzt, besonders scharf, wenn die Stränge dünn sind, oder wenn sie durch hineingepresste oder bineingewucherte Zellen des Nacbbargebietes mebr oder weniger unregelmässig geworden sind. Nicht ganz selten stiess ich auf bleine Blutgefässe (Arterie oder Vene), deren Lumen mit einigen. Reiben rother Blut-Körperchen gefüllt ist, deren Endothelien ganz normal aussehen, während die ganze übrige Gefåsswand, wenn auch normal dick, hyalin degenerirt ist und von einer Anhäufung gewucherter Zellen begleitet wird. Auf diese Zellen komme ich nochmals zuräck. In den peripherischen Partien, sowohl an der atrophischen Musculatur, als auch unter der entzündlich verdickten Ilaut, wo massenhaft Geschwulstzellen zu finden sind, treten byaline, sehr dünne Stränge entweder gar nicht auf, oder nur in geringer Menge. Wenn das letztere der Fall ist, zeigen die Stränge eine plexiforme Anordnung, welehe einem Gefässnetz entspricht, oder es lassen sich längliche, feine Stränge als hyalin degenerirte Bindegewebsfasern nacbweisen. Manchmal sind die Stränge nicht so deutlicb byalin, sondern von feinkörniger Structur.

In einigen Präparaten, welche ich an der Grenze der Musculatur entnommen habe, finde ich Reste von Muskelfasern, welche, im Gegensatz zu den benachbarten stark atrophischen Muskelfasern, sehr aufgequollen erschienen, ihre Querstreifung verloren, und ein feinkörniges Aussehen darbieten; die meisten. Muskelkerne sind verschwunden, abgeplattete Kerne dicht am Sarcolemma nur vereinzelt vorhanden. Solcbe Reste von Muskelfasern von nur geringer Länge bleiben neben anderen atrophischęn Muskelfasern 
vereinzelt zwischen den Geschwulstzellen sichtbar. Je tiefer in der Geschwulst ein solcber Muskelrest bestehen geblieben ist, desto byaliner erscheinen seine Fasern, so dass man sie von andern hyalinen Strängen dem Ausseben und der Beschaffenheit nach kaum unterscheiden kann. Indem man den Uebergang von solchen byalinen ususelresten bis in das unbestreitbare Muskelgewebe hinein verfolgt, kann man ihren musculösen Ursprung erkennen. Diese Umwandlung und Betheiligung atrophischer Muskelfasern an der Eyalin-Bildung erscheint freilich in meinem Fall ziemlicb selten. Die byalinen Kugeln, wie sie Marchand') und v. Ohlen ${ }^{2}$ ) in ihren Fällen beschrieben baben, sind von mir nicht nach: gewiesen worden.

Noch wichtiger und interessanter, als diese hyalinen Gebilde, sind die zelligen Elemente, welche ein ziemlich complicirtes Verbalten zeigen. Je nach der Stelle, wo sie sich vorfinden, sind die Zellen sebr verschieden gestaltet und angeordnet. An manchen Stellen lagern die Zellbaufen neben den byalinen Strängen,- und bilden, ebenso wie diese selbst, allerlei anastomosirende Zapfen, Stränge oder Netzwerke. Besonders in der centralen Partie der Geschwulst bestehen sie aus kurzspindeligen, bauchigen, ovalen und sogar runden, dicht aneinander liegenden Zellen, deren Kerne gross, chromatinreich und gewöhnlich der Zellform angepasst sind. Die Gestalt, und besonders die Anordnung der Zellstränge, Jassen an manchen Stellen wit voller Sicherheit nachweisen, dass sie als gewucherte Endothelien der Lymphgefässe aufzufassen sind.

Wie ich schon oben angedeutet habe, findet sich die zellige Anbäufung um einzelne Blutgefässe, die mit normal dicker, aber byalin degenerirte: Wandung versehen sind. Diese zelligen Elemente verhalten sich genau wie die anderen, nur sind diese zelligen Stränge manchmal zu besonderer Dicke gediehen. Das Bild erinnert an die Geschwulst-Art, die von manchen Autoren als "Angiosarcoma" oder" "Perithelioma" bezeichnet wird. An einigen Stellen ist aber sehr klar zu constatiren, dass es sich um nichts anderes, als um gewucherte Endothelien präformirter, extravasculärer Lymphgefässe, bezw. Lymphräume handelt; die Zellstränge umgeben das Blutgefäss wie ein Mantel, und man kann noch deutlich die Wand und die Wand-Elemente der Lymphgefässe nachweisen. In anderen Fällen sind die Zellen weiter in das Gewebe hineingewachsen, so dass eine scharfe Grenze gegen die Nachbarschaft nicht mehr vorhanden ist; dadurch tritt das eigenthümliche Verbalten des Endotbelioms nicht mehr klar hervor. Kurz, das ganze Bild ergiebt deutlicb eine Endothel-Wucherung der Lymphgefässe, bei gleichzeitiger hyaliner Degeneration der Gerüstsubstanz. Die Grenze der gewucherten Endothelien gegen die letztere ist entweder ganz glatt und scharf, oder un-

1) Marchand: Ueber ein Endotheliom mit hyalinen Kugeln des Antrum Highmori. Ziegler's Beiträge Bd. XIII, S. 479.

2) v. Ohlen: Beitrag zur Kenntniss der Parotis. Geschwülste. Ziegler's Beiträge XIII, S. 456. 
regelmässig, je nachdem die Zellen weiter zur. Seite gewuchert sind, oder die hyaline Substanz zwischen die Endothelien eindringt.

An anderen Stellen, sowohl im Centrum, als insbesondere an der Peripherie, ist das Bild insofern ein anderes, als die hyaline Degeneration an der Gerüstsubstanz fast gar nicht, oder nur in sehr geringem Grade eingetreten ist. Neben dem reinen Endothel-Gewebe werden massenhaft Bündel von spindelförmigen Zollen wahrgenommen. Diese Bündel durchflechten einander, so dass - sowohl ihrer Stärke, als ibrer Richtung nach - das Bild demjenigen eines Uterusmyoms ähnlich wird, and das characteristische Verbalten der Spindelzellen-Sarcome zu Tage tritt. Gestalt und Grösse der Spindelzellen sind nicht immer gleich. Bald herrscht die kurze, bauchige Spindelform vor, bald besteht das Bündel aus langen, bandartigen Zellen. Tebergangsformen zwischen beiden sind aber auch vorhanden. Diesë Spindelzellen lassen sich einerseits bis zwischen die atrophischen Muskelfasern verfolgen, andererseits berühren sie direct den oben erwähnten endotheliomatōsen Abschnitt wit der hyalinen Degeneration. Unterscheiden sich die beiden Geschwulst-Elemente durch ihre Form und besonders durch ibre Anordnung deutlich voneinander, so findet doch stellenweise der Uebergang ganz allmählich statt, und das Bild wird dadurch sehr verwickelt. An wenigen Stellen, besonders in den Präparaten, welche ich aus einer zellreichen Partie senkrecht gegen die Muskelfasern angefertigt bahe, sehe ich eine Anzahl der kleinen und grösseren Alvealen, dicht nebeneinander liegend; ibr Wandgang ist schmal und besteht aus faserigen, bezw. mehr oder weniger byalin degenerirten Bindegewebsfasern; die Maschenräume sind mit Zellen gefüllt, von denen die wandständigen immer klein, kugelig und mit intensiver tingirtem Kerne versehen erscheinen, wäbrend die in der Mitte gelegenen Zellen grōsser und vielgestaltiger sind, und hlasser gefärbte Kerne besitzen. Ist dieses ganze Bild auch dem des sogenannten Alveolar-Sareoms sebr äbulich, so bemerkt man doch bei genauerem Studium sofort, dass die Inhaltsmasse jedes Alveolus aus einem Bündel spindelförmiger Zellen besteht, welche quergeschnitten sind, dass dagegen in dem schmalen Alveolar-Septum kleine, rundliche, mit stärker gefärbtem Kerne versehene Zellen, theils junge Bindegewebszellen, theils Wanderzellen, lagern. Hier und da tritt eine circumscripte Nekrose in geringer Ausdehnung mitten in der zellreichen Partie auf. Ausserdem erfabren, besonders in der Năhe der ausgedehnten byalinen Heerde, einige Bündel langer Spindelzellen eine eigentbümliche Umwandlung. Zuerst wird der Zellkern blasser und undeutlicher, das Protoplasma immer mehr homogen, der Contur einzelner Zellen verwischt, bis endlicb das ganze Bündel der byalinen Entartung verfalle $n$ ist. Diese Art der Hyalin-Bildung kommt in dieser Geschwulst nicht oft vor, und bleibt dann immer auf kleine Strecken beschränkt.

Ist der bisher geschilderte Befund an und für sich schon interessant, so liegt doch die hauptsächliche Merkwürdigkeit dieses 'Tumors in dem Vorhandensein jener eigenthümlichen Zellen, welche ihn als "Rhabdom yo- 
sarcoma“ oder "Myosarcoma striocellulare" bezeicbnen lassen. An der Stelle, wo sicb das Bild des Spindelzellen-Sarcoms darstellte, traf ich ganz zufällig in einigen Präparaten bald ziemlich massenhaft, bald nur spärlich Zellen mit einer sehr deutlichen Längsstreifung; andererseits enthielten die Bündel des Spindelzellen-Sarcoms, wie oben schon angedentet, Zellen wit deutlicber Querstreifung. Diese langgestalteten Zellen sind entweder bandartig gestreckt, oder sie übertreffen in ibrer Länge die gewöhnlichen spindeligen Sarcomzellen nicht, können sogar noch kürzer als diese erscheinen. Die Zellen, bezw. Fasern sind meistens schmal, baum den doppelten Breitendurchmesser eines.Kernes überschreitend. Der letztere ist immer lang, oval, granulirt and chromatiureich, 1-2 Kernkörpercben enthaltend. Manchmal ist die Kern-Granulation in punctirten, parallelen Linien angeordnet, so dass bei flüchtiger Untersuchung eine Querstreifung vorzuliegen scheint. Die Beschaffenheit der einzelnen Zellen ist am besten im sorgfältig gemachten Zupfpräparate zu erkennen. Man sieht aber verschiedene Formen dieser Zellen. Manchmal ist die Zelle bandartig und ziemlich lang, in einer starken Vergrösserung über zwei Gesichtsfelder reichend $(600-620-b 50 \mu$ ). Die Breite des Bandes bleibt gewöhnlich in seiner ganzen Länge, abgeseben von den beiden Enden, dieselbe. Der längsovale Kern sitzt entweder mitten in der Zelle, welche eine entsprechende spindelige Auftreibung trägt, oder er erscheint nur sejtlicb angelagert, gleichsam angeheftet, wie es Marchand in seinem Falle von Myosarcom. schon beschrieben und besonders betont hat. Wenn die Faser dick ist, so bildet. sie keine Auftreibung an der Kernstelle. Die Kerne solcher Zellen sind gewöbnlich in einer einzigen Reibe angeordnet, aber man sieht auch Gruppen von 3-5 Kernen an dem ausgebauchten Theile einer Faser.

Die Querstreifen, d. h. abwechselnd dunkle und helle scharfe Stricbe, sind gewöhnlich ganz senkrecht gegen die Zellachse gerichtet, und verlaufen entweder in einer geraden, oder aber in einer lejcht gebogenen Linie. Sie Lann ebenso deutlicb in den schmalen, wie in den dickeren Zellen erscheinen. Meistentheils tritt Querstreifung in der ganzen Lānge des Bandes überall deutljch zu Tage; bei einzelnen Zellen ist dieselbe aber nur neben dem Kerne auf eine kurze Strecke bin deutlich, andererseits nur in der peripherischen Zone der Faser wahrnebmbar, wäbrend die directe Umgebung der Kerne nur leicht körnig erscheint. Ob eine solche Faserzelle am äusseren Umriss wit irgend einem Häutchen versehen ist, das iusst sich sehwer entscheiden. Bei wohl ausgebildeten dicken Fasern scheint ein solcbes regelmässig ausgebildet zu sein. Ihre Enden sind entweder scharf zugespitzt, oder kolbig aufgetrieben und frei von Querstreifung, während dann eine Längsstreifung ihnen zukommen kann. Ausser dieser langen, bandartigen Gestalt bieten die mit Querstreifung versehenen Zellen noch andere Formen dar, so z. B. die Spindelform. Langspindelige Zellen mit Querstreifung und Längsstreifung finden sich sogar recht häufig, entweder bi- oder unipolar; der Kern kann alsdann grösser sein, wie an den langen, bandartigen Zellen, 
meist einfach, selten doppelt, entweder in der Mitte der Zelle, oder am kolbigen Ende gelegen. Die Querstreifung erscheint in solchen längeren Zellen im Allgemeinen weniger deutlich, als bei der bandartigen Form. Manchmal ist die Querstreifung nur auf gewisse Stellen der Zelle beschränkt, ist entweder neben dem Kerne, oder nur am Ende, oder nur am peripherischen Rande der Zelle deutlich ausgeprägt, während die die Kerne umgebende Substanz nur leicht körnig oder höchstens längsgestreift erscheint, wie es bei der Faserform zuweilen der Fall war. Es giebt auch Zellen von ganz kurzspindeliger bauchiger Form, in deren Protoplasma die Quer- und Längsstreifung nur undeutlich auftritt. Die wohl ausgebildete, lange, quergestreifte Faser färbt sich mit Fuchsin-Pikrinsäure dunkelgelblich, wie die gewöhnliche Muskelfaser. Glycogen konnte bei wiederholter Untersuchung nicht nacbgewiesen werden.

Wichtig ist die A nordnung solcher langen und kurzen Zellem mit Querstreif ung. Sie kommen entweder in ziemlicb beträchtlicher Menge neben einander, oder vereinzelt zwischen ähnlich gestalteten Spindelzellen vor, welche selbst der Streifung entbehren. Ueberhaupt aber ist die Querstreifung ziemlich selten, und man muss recht viele Präparate anfertigen, um ein günstjges zu gewinneu. Wo aber auch die quergestreiften Zellen, bezw. Fasern reichlich auftreten, da sind sie doch immer mit längeren oder kürzeren, spindelförmigen, ungestreiften Zellen gemischt. Die Kerne der letzteren sind immer ähnlich denjenigen der quergestreiften Zellen, nur etwas stärker gefärbt. An einigen Stellen erscheinen $3-5$ solcbe quergestreifte Zellen, hezw. Fasern dicht neben einander aufgestellt, ohne dass dazwischen spindelförmige Sarcomzellen vorbanden sind. Indem hier diese Zellen und Fasern von Bündeln massenhafter Spindelzellen umschlossen werden, entspricht ihr. Verlauf immer der Richtung dieser letateren; bald sind sie parallel den gesunden Muskelfasern, welche man weit ab ander Grenze der Geschwulst wahrnimmt, angeordnet, bald kreuzen sie die Richtung der Muskelfasern. Was den Ort anbetrifft, wo solche Fasern und Zellen vorkommen, so begegnet man ihnen immer im Innern eines Bündels von spindelzelligen Sarcom-Elementen; dagegen findet man sie gewöbnlich nicht da, wo die hyalinen Stränge und das Endothelion deutlich erscheinen. Wenn aber die letzteren Geschwulst-Abschnitte direct an den spindelzelligen Theil anstossen, finden sich vereinzelte Zellen und Fasern mit Querstreifung auch hier vor, unter Umständen auch hart neben den byalinen Strängen. Irgend eine anatomische Beziebung der quergestreiften Zellen za den Endotbeliomzellen ist nicht vorhanden. Wo die Spindelzellen-Bündel ganz an der Peripherie der Geschwulst aufgetreten sind und sich direct mit den atrophiscben Muskelfasern berühren, kommen die Zellen und Fasern mit. der Querstreifung nicht zum Vorschein, vielmehr immer erst in den inneren Partien der Geschwulst.

Durch genaue Untersuchung recht vieler Präparate habe ich festgestellt, dass: die quergestreiften Fasern und Zellen nichts mit den präformirten 
Muskelfasern zu thun haben, wenigstens nicht hinsichtlich ihrer Aufstellung. Manchmal trifit man zwischen den quergestreiften Zellen oder den Spindelzellen ohne Querstreifung runde Gebilde, welche einen kaum 2-3 fach grösseren Durchwesser haben, als die anderen Zellea, und lejcht punctirt oder gestreift sind. Häufig werden 1-3 Kerne darin gefunden. Bier ist entweder ein kolbig aufgetriebenes Ende einer langgestreckten Faser quergescbnitten, oder die Mitte einer wobl ausgebildeten quergestreiften Faser getroffen. Wenn der Schnitt etwas schräg trifft, so sieht man genau dasjenige Gebilde, welches man "Muskelknospe“ genanot hat, indem ein rundkolbiges, kernhaltiges Gebilde in einen schmalen Stiel übergeht.

Ich darf einige Bemerkungen über den ganz peripherischen Theil der Geschwulst nicht unterlassen. Die Geschwulstzellen dringen daselbst zwischen die atropbischen, aber deutlich quergestreiften Muskelfasern ein, welche meist der Kernfärbung verlustig gegangen sind. Diese Geschwulstzellen sind meist klein, kurz, schmal und spindelförmig, mit einem gutgefärbten, längsovalen Kern versehen. Sie ordnen sich in der nächsten Nähe und in der Richtung der Muskelfasern parallel an einander an, während in einiger Entfernung schon deutliche Bündel in verschiedenen Richtungen verlaufen. Die grosse und lange Form der. Spindelzellen bommt hier eben so wenig vor, wie die.Quer- und Längsstreifung. Eine byaline Degeneration am Gerüst ist gar nicht nachweisbar, so dass man höchstens Zellen findet, an denen sie wirklich, immer aber nur in gani schwachem Grade aufgetreten ist.

Was die Veränderungen der Muskelfasern an der Grenze gegen die Geschwulstmasse hin betrifft, so werde ich sie in einer anderen Arbeit äbersichtlich darstellen, und jch brauche daher hier in das Detail nicht genauer einzugehen. Nur auf eins mache ich aufmerksam; dass die Muskelfasern an der Peripherie der Geschwulst immer sich passiv verhalten und alle die einfache Atrophie, welche durch die ins Perinupium int. eindringenden Geschwulstzellen verursacht wird, zeigen. Wie gesagt, sind die Muskelkerne nicht gut tingirbar; irgend ein activer Vorgang in der Muskelfaser, wie Kernvermehrung, deren'Bedeutung verschieden, sowohl im progressiven, als auch im regressiven Sinne aufgeklärt wird, ist nirgends nachweisbar.

An der Haut zeigen die Geschwulstzellen, falls sie bis unter dieselhe vordringen, das gleiche Verhalten, und ich sebe die gleichen Geschwulstzellen in das Unterbaut-Fettgewebe fortschreiten. Zwischen den einzelnen Fettzellen sind viele kleine, spindelige oder mehr rundliche Zellen in wechselnder Menge wahrzunebmen. An anderen Stellen der Haut, besonders an der vernarbten Stelle und an der dicht nebenan liegenden buckeligen Hervorragung, traf ich eine gewisse Variation in der Anordnung der Geschwulstzellen. Dort in der Narbe sind die Geschwulst-Elemente, sowobl die kleinen Spindelzellen, als auch die darin eingebetteten rundlicben oder ovalen Zellen, nehmlich gewuoherte fixe Bindegewebszellen, : Wanderzellen und stellenweise gewucherte Iymphgefüss-Endothelien, direct durch" ein verdicktes, faseriges Bindegewebe abgegrenzt, ebenso, wie an den Stellen:der Subcutis, In daș 
tiefere Bindegewebe setzen sich einige schmale Züge von Geschwulstzelten continuirlich fort, besonders die Blutgefässe entlang. Ausserdem beobachtet man in ihm hie und da eine geringe Rundzellen-Infiltration, und zuwoiten sieht man die mehr rundlichen und kleinen Zellen in der Ueberzahl.

Die buckelig hervorragenden Geschwulstmassen, welche sich aussen an der Baut neben der Narbe hefinden, besitzen an der peripherischen Partie noch eine epitheliale Bekleidung, während der grösste mittlere Theil derselben entbehrt. Hier besteht eine schmale, oberflächlichste Schicht aus einer nekrotischen, körnigen, structurlosen Masse, in welche kleine Rundzellen (Leukocyten) in variabler Menge eingebettet sind. Unter dieser Schicht treten massenbaft Zellen auf, ohne irgend eine scharf gezogene Grenze, Diese Zellen sind meist rundlich, oder kurz spindelig oder oval; mit kleinem, gut tingirtem Kerne versehen; ibre Anordnung wird durch das bindegewebig-faserige Gerüst mit Blutgefässen bestimmt. Man kann immer constatiren, dass die dicht an diesem bindegewebigen Gerust liegenden Zellen die jüngeren, und dass die anderen von diesen ausgegangen sind. Wenn man die Geschwulst von aussen nach innen verfolgt, so sieht man die Zellen länglicher und deutlicher spindelig werden, wäbrend im Princip das Bild dasselbe bleibt, wie unter der Haut und in der pheripberischen Partie der Geschwulst. Es handelt sich nur um eine gewisse Modification der Zellform. Auch in den peripheriscben buckeligen Geschwulsttheilen ist eine byaline Degeneration am Bindegewebsgerüst und an der Gefässwand nachzuweisen, freilich nicht so ausgesprägt, wie im centralen Theil der Geschwulst; auch sind hier die byalinen Stränge nur schmal. An einigen Stellen der oberflächlichen Schicht trifft man ein eigenthümliches Bild der hyalinen Degeneration. Man sieht z. B. die Wandung eines Blutgefässes vollkommen hyalin umgewandelt und um dieses Gefäss herum eine Menge kleiner aber vollkommen byaliner Klumpen von verschiedener Gestalt und Grösse, welche dicht zusammengedrängt sind. Nicht selten ist an solcben Kluwpen kein einziger Kern mebr vorhanden, oder nur ein vereinzelter atrophischer. Durch genauen Vergleich wit der wenig veränderten Nachbarschaft erkenut man, dass es sich um eine hyaline Degeneration der Zellen und Bindegewebsfasern handelt. Bie und da sind Anbäufungen von rothen Blutkörperchen aufgetreten, sowohl in präformirten Canälcben, besonders an erweiterten Lymphbahnen, als auch im Gewebe selbst zwischen den Geschwulstzellen. Eine solche Stelle ist schon makroskopisch durcb eine bräunlich-rothe Farbe gekennzeichnet. Die meisten rothen Blutkörperchen sind wobl erhalten, dagegen an einigen Stellen körnig zerfallen; aucb feine Fibrinfäden ziehen durcheinander und sind durch die Weigert'sche Reaction zu charakterisiren. Nicht ganz selten werden vereinzelte hyaline Klumpen und Fasern dazwischen beobachtet. Ich babe auch in wenigen Fălen einen Venendurchschnitt gesehen, welcher mit hyaliner Masse vollgestopft war.

Der geschilderte Tumor setzt sich also der mikroskopischen Untersuchung nach aus verschiedenen Elementen zusammen, 
bald liegt eine Wucherung der Endothelien der Lymphgefässe vor, bald sind spindelige Sarcomzellen aufgetreten. An vielen Stellen sind beide Elemente nebeneinander vorhanden, so dass das Bild sich sehr verwickelt, und manchmal eine Grenze $z$ wischen beiden kaum gezogen werden kann. Oft beobachtet man auch neben den zelligen Elementen ein ausgedehntes Auftreten hyaliner Zapfen, Stränge und Kolben, welche verschiedene Richtungen einhalten, meist aber plexiforme Anordnung zeigen. Das Bild entspricht alsdann dem sogenannten "Cylindrom" Billroth's, und die von ihm gegebene Definition passt ganz gut auch auf dieșen Fall.

Ueber diese Bezeichnung ist ja viel discutirt, und mehr als zehn verschiedene Namen sind vorgeschlagen worden. Nach Sattler ${ }^{1}$ ) haben noch Kolaczek ${ }^{2}$ ), Frank ${ }^{3}$ ) freilich vergeblich versucht, die so complicirten Geschwülste in eine einheitliche Gruppe zu bringen und einen besonderen Namen dafür zu wählen. Da aber die hyaline Degeveration bei verschiedenartigen Geschwülsten auftritt und nichts Einheitliches bezeichnet, so hat Lubarsch ${ }^{4}$ ) mit einem gewissen Recht vorgeschlagen, nicht einfach "Cylindrom" zu sagen, sondern die Bezeichnung "Sarcoma, Carcinoma, oder Endothelioma cylindromatosum" zu gebrauchen. Ich stimme diesem Vorschlag deswegen bei, weil in meinem Falle die zelligen Elemente hauptsächlich zweierlei Ursprungs sind, und weil die hyaline Substanz durch die Umwandlung verschiedenartiger Elemente zu Stande kommt. Die Geschwulst mit hyaliner Degeneration, - nennen wir sie kurzweg Cylindrom - , ist bekanntlich in verschiedenen Körpertheilen und in mancherlei Organen beobachtet worden. Dennoch fand ich, so viel ich auch die bis jetzt in der Literatur angegebenen, die Zahl 50 übersteigenden Fälle von Cylindrom und

1) Sattler, (Sarcoma carcinomatosum). Ueber die sogen. Gylindrome und deren Stellung im onkolog. Systeme. Berlin, 1874.

2) Kolaczek, (Angiosarcom). Ueber das Angiosarcom. Deutsche Zeitschrift für Chirurgie. Bd. 9, S. 10, 165, Bd. 13, S. 1.

3) Frank, (Endothelioma intravasculare hyalogenes). Beiträge zur Geschwulstlehre. Dieses Arch., Bd. CXXI, S. 465.

4) Lubarsch, Ueber die Geschwulst-Bezeichnung "Cylindrom". Dieses Arch., Bd. CXXII, S. 573. Ergebniss der allgem. pathol. Morphologie und Physiologie, 1895, S. 369. 
verwandten Tumoren vergleichen konote, keinen, welcher sich an analoger Stelle, nehmlich in der Körper-Husculatur, ausgebildet hatte.

Ueber die Herkunft der hyalinen Substanz sind die Ansichten getheilt. Sobald eine plexiforme Anordnung derselben hervortrat, und die Blutgefässe, bezw. die Säulen der Blutkörperchen mitten in den hyalinen Strängen erschienen, waren die Autoren meistens geneigt, die hyalinen Stränge als degenerirte Blutgefässwände aufzufassen. Während die Einen (v. Recklinghausen ${ }^{1}$ ), Kocher ${ }^{2}$ ), Förster ${ }^{3}$ ), Billroth ${ }^{4}$, Oeller ${ }^{5}$ ) u. s. w.) diese Veränderung der $W$ andung deutlich blutgefüllter Gefässe beobachtet haben, konnten in anderen Fällen (z. B. Friedreich ${ }^{6}$ ), Köster ${ }^{7}$ ) Blutgefässe nicht nachgewiesen werden. In meinem Fall ist ein Blutgefäss im Innero des hyalinen Gebildes auch nur ein inconstanter Befund; dennoch lassen sich die meisten solcher plexiformen Stränge als hyalin degenerirte Wandungen der Blutgefässe, sowohl der Capillaren, als auch der kleineren Arterien und Venen bestimmen. Die hyaline Substanz quilit auf und nimmt an Volumen derart zu, dass sich das Lumen der Capillaren gänzlich verschliesst. Dabei zeigt sie manchmal gegen die benachbarten Zellen hin eine unregelmässige Begrenzung; die Zeichnung kann sogar ungemein mannigfaltig werden dadurch, dass auch diese wuchernden Zellen $z$ wischen die hyalinen Stränge eingepresst, and in sehr wechselnde Gestalten umgeformt

1) v. Recklinghausen, (Gräfe, zur Casuistik der 'Tumoren). Gräfe's Arch. f. Ophthalmologie, Bd. X, Abtbeilung 1, 1864, S. 184.

2) Tb. Kocher, zur Kenntniss der pulsirenden Knochen-Geschwülste nebst Bemerkung über byaline Degeneration. Dies. Arch., XLIV, S. $311,1868$.

3) Billroth, Untersuchungen über Entwickelung der Blutgefässe u. s. w. Berlin, 1865.

4) Förster, Atlas der mikroskopischen Anatomie. Leipzig, 1854-1859. Tafel XXX.

5) Oeller, Ueber hyaline Gefäss-Degeneration als Ursache der Amblyopia saturnina. Dies. Arch., LXXXVI, S. 329, 1881.

6) Friedreich, zur Casuistik der Neubildung. Dieses Arch., XXVII, S. 375,1863 .

万) Köster, Cancroid mit hyaliner Degeneration. Dies. Arch., Bd. XL, S. $468,1867$. 
werden. Beachtet man aber, dass die plexiform geordnete hyaline Substanz ein continuirliches Netzwerk bildet, so muss man zweifellos die Balken desselben als Gefässwandungen anerkennen, und das Vorkommen der rothen Blutkörperchen inmitten des einzelnen hyalinen Balkens macht es zur unumstösslichen Thatsache. Dazu gesellt sich noch eine hyaline Degeneration der Bindegewebsfasern and der Geschwulst-Elemente selbst. Unter den letzteren erscheinen spindelförmige Sarcom. zellen mit Sicherheit betheiligt, and vielleicht auch endotheliomatöse Zellen. Ihre Umwandlung in Hyalin geht in meinem Fall ganz allmäblich und gleichmässig am ganzen Zellenleib vor sich, nicht unter Vacuolenbildung partieller HyalinAblagerung innerhalb der Zellen, wie es $v . O h l e n^{1}$ ) in seinem Fall beobachtet hat.

Dass an einzeinen Stellen auch eine hyaline Umwandlung der Muskelfasern vorkommt, halte ich für sehr wabrscheinlich. Diese Betheiligung der Muskelfasern hat aber nur eine nebensächliche Bedeutung und ist nicht ausgesprochen, wie es Lü $\mathrm{cke}^{2}$ ) hinstellte, indem er bei- einem Cylindrom der Zunge die hyaline Substanz auf. eine Degeneration des Zungen-Muskels zurückzuführen versuchte. Ebenso ist eine Entstehung des Hyalins aus alten Blut-Bestandtheilen in meinem Fall kaum in Anschlag zu bringen. Was die Natur der hyalinen Substanz betrifft, so bietet sie immer dieselben chemischen Eigenschaften dar. Es versagt hier jede Reaction auf Mucin, welches häufig mit Cylindrom combinirt vorkommt, and sich alsdann meistens gegen das Hyalin auch nicht scharf abgrenzen lässt. Eine amyloide Degeneration, auf deren Vorkommen besonders Lubarsch ${ }^{3}$ ) aufmerksam machte, ist hier ebenfalls nicht zu Stande gekommen.

Ich kann daher behaupten, dass in meinem Fall die Natur der hyalinen Substanz eine einheitliche ist, trotz ihres Ursprungs aus verschiedenen Elementen des Gewebes, dass aber den Elementen des Tumors in ganz besonderem Maasse die Tendenz innewohnt, sich byalin umzuwandeln.

1) จ. Oblen, a. a. 0 .

2) L ü cke, 'Beiträge zur Gesehwulstlehre. Dies. Arch., XXXV, S. 520, 1866.

3) Lubarsch; a. a. 0 . 
Dass die zelligen Elemente. dieses Tumors einerseits als Spindelzellen, andererseits als Endothelzellen der Lymphbahnen zu bezeichnen sind, habe ich schon erläntert. Weiter fragt es sich nur, was sind jene nit Querstreifung versehenen Zellen und Fasern? Ein derartiges Gebilde erscheint dem Aussehen: nach äusserst ähnlich, ja identisch mit einer sich noch im Stadium der embryonalen Entwickelung befindenden quergestreiften Muskelfaser und entspricht in den meisten Punkten auch den Schilderungen und Abbildungen des. Rhabdomyosarkoms seitens der oben erwähnten Autoren. Eine solche Zelle stimmt auch ganz mit den quergestreiften Faserzellen eines Rhabdomyosarcoms des Hodens überein, welches ich selbst vergleichen konnte. Man könnte freilich denken, dass es sich um atrophische Muskelfasern handele, welche zwischen den Geschwulstzellen zurückgeblieben sind. Deutlich sind ja an dem peripherischen Theil dieser Geschwulst einfach atrophische Muskelfasern nachzuweisen, selbst in grosser Menge, und diese schmalen Muskelfasern sind den langen, quergestreiften Fasern mitten in der Geschwulst der Form nach ziemlich ähnlich.

Die Frage ist also, ob die quergestreiften Fasern und Zellen der Geschwulst nur atrophirte Muskelfasern sind (wie frühere Autoren schon behauptet haben), oder ob hier eine Neubildung vorliegt. In meinem Fall bin ich berechtigt, letztere Auffassung für die zutreffende zu erklären, und zwar ausser der morphologischen Beschaffenheit der Zellen und Fasern selbst noch aus folgenden Gründen: 1) in Rücksicht auf die Lage: Die quergestreiften Zellen und Fasern finden sich nie an der äussersten Peripherie des Tumors, sondern etwas mehr im Innern, da, wo die atrophischen Muskelfasern nicht mehr vorkommen; 2) in Rücksicht auf die Anordnung: Sie sind in die Bündel der spindelförmigen Sarcomzellen eingebettet und verlaufen in der Richtung der letzteren, d. h. manchmal in einer die gesunden Muskelfaser kreuzenden Richtung; ferner treten sie manchmal dicht nebeneinander zusammenliegend auf; dagegen sind die atrophischen Muskelfasern in der peripherischen Zone der Geschwulst immer durch eindringende Geschwulstzellen gesondert, und daher vereinzelt gelegen; 3) lassen sich die verschiedenen Stufen des 
Wachsthums von kürzeren Spindel- bis zu den langen Faserzellen verfolgen; 4) sind ihre Kerne deutlich zu färben, während die atrophischen Muskelfasern der Geschwulst-Peripherie ihre Kernfärbung meist verloren haben, oder Kerne besitzen, die nur ganz schwach gefärbt und viel kleiner sind; 5) kommt nicht minder die Evidenz des Uebergangs zu den sonstigen nahestehenden Spindelzellen, also typischen Sarcomzellen, welche weder Quer- noch Längsstreifung besitzen, in Betracht.

Eine weitere Frage ist die nach der Natur und Herkunft dieser quergestreiften Zellen, ob sie nehmlich wirklich musculöser Natur sind oder nicht, ob sie eine besondere Stellung gegenüber den gewöhnlichen spindelförmigen Sarcomzellen einvehmen, oder ob sie nur als eine Modification der letzteren $z$ u betrachten sind. In Bezug hierauf seien für meine Geschwulst folgende thatsächlichen Momente hervorgehoben: 1) Die Aehnlichkeit der Zellen und Fasern mit jungen embryonalen Muskelfasern einer gewissen Entwicklungszeit. 2) Die Uebereinstimmung meiner Angaben mit den Schilderungen und Abbildungen der Zell-Elemente des "Rhabdomyoma", wie sie in den Werken anderer zuverlässiger Untersucher enthalten sind, hier zu nennen: Marchand ${ }^{1}$ ) Tafel IV. Fig. 1. k. p. n., Ribbert ${ }^{2}$ ) Taf. VII. Fig. 1. rechts, 9. 14. 3, ein Theil von 8, Wolfensberge ${ }^{3}$ ) Tafel X. Fig. 9. 20.13. 3. Rundliche Gebilde mit oder obne Streifung, denen man in den angeführten Arbeiten dieselbe (musculäre) Herkunft zuschreibt, sind in meiner Geschwulst nicht aufgefunden worden, nur einige dickbauchige Zellen mit einer wenjger deutlichen, offenbar modificirten Quer- oder Längsstreifung; immer aber war die spindelige Gestalt noch gut ausgeprägt. Auf den Glycogengehalt solcher Geschwulstzellen hat zuerst Marchand die Aufmerksamkeit gelenkt, in Rücksicht darauf, dass der centrale Theil der jungen Muskelfasern einer bestimmten embryonalen Entwicklungs-Periode granulirt erscheint und mit einer beträchtlichen Menge von Glycogen durchsetzt ist. Da aber das Glycogen leicht in Wasser löslich ist, kann die Faser in ihrem Innern auch ein helles Aussehen darbieten, anscheinend sogar

1) Marchand, a. a. O. Dieses Archiv C.

2) Ribbert, a. a. 0. Dieses Archiv CXXX.

3) Wolfensberger a. a. 0 . 
eine centrale Lücke enthalten ${ }^{1}$ ). Die Zellen und Fasern in meinem Fall sind aber meistens ganz solide und weisen nur in sehr kleiner Anzahl die centrale Höhlung auf; die Reaction auf Glycogen bleibt immer negativ, trotz aller möglichen Modificationen derselben. Freilich war das Präparat schon jahrelang in Spiritus aufbewahrt worden; aber schon bei der wiederholten Probe im frischen Zustande hatte die Lugol'sche Lösung Herrn Prof. von Recklinghausen immer nur ein negatives Resultat ergeben. Laut den Angaben auch anderer Autoren wurde das Glycogen in rhabdmyomatösen Geschwülsten nicht immer nachgewiesen.

Da nun die Querstreifung als ein Charakteristicum für die Muskelfaser anzusehen ist, insofern sie in keinem anderen Gewebs-Elemente zu constatiren ist, so denke ich, dass die vorliegenden Gebilde wenigstens im morphologischen Sinne als "Muskelfaser" bezeichnet werden dürfen, obwohl die übrigen biologischen Eigenschaften z. B. die Contractilität und die Doppelbrechung nicht constatirt worden sind. Der Nachweis der ersteren ist unmöglich bei dom gehärteten. Präparat, und die letztere finde ich mit Marchand unnöthig. In diesem Sinne nehme ich die Bezeichnung "Rhabdomyosarcom" an, obne aber damit unüberlegt aussprechen zu wollen, dass diese Faser ganz ebenso, wie die richtige quergestreifte Muskelfaser entstanden sein muss. Hinsichtlich der an Spindelzellen beobachteten Streifung hat schon Virchow ${ }^{2}$ ) in seinem classischen Werke die Möglichkeit hervorgehoben, dass Spindelzellen längsstreifig und auch mehr oder weniger querstreifig werden können. Solche Zellen erscheinen "wie quergestreift, jedoch so, dass die Querstreifungen nicht die gleichmässigen, continuirlichen Varicositäten bilden ${ }^{3}$ )." In meinem Fall aber sind die Querstreifungen so deutlich und so regelmässig aufgetreten, dass diese Spindelzellen dem Aussehen nach mit jungen Muskelfasern zu identificiren sind. Die Querstreifen erscheinen als ununterbrochene Linien in gleichmässigen Abständen von einander; sie nehmen in den Spindel-

1) Ranvier, Traité technique d'histologie 1875, p. 513.

?) Virchow, die krankhaften Geschwülste, Zweiter Band. S. 198. $1864-1865$.

3) Virchow, ebendaselbst, S: 199., (Würzburgẹ Verbandlung Bd. I. 1850. S. 190.) 
zellen eine sehr grosse Strecke ein und treten, wo sie vorhanden, immer in relativ zahlreichen Zellen und Fasern auf. Ich meine daher, in meinem Falle handele es sich um etwas anderes, als in der Virehow'schen Beschreibung. Auch die von Virchow erwogene Möglichkeit, „ob nicht die Querstreifang. der Muskeln nur die stärkere Entwickelung einer Eigenschaft sei, welche auch anderen faserigen Elementen zukommt und nichts Specifisches an sich hat $\left.{ }^{1}\right)^{\text {, }}$, hat bis jetzt Niemand in positiver Weise bestätigt und auf eine reale Unterlage gestellt.

Darf die Genese unserer neugebildeten "Muskelfasern" der bei den anderen Rhabdomyosarcomen behaupteten Herkunft gleichgesetzt werden?. Die meisten Autoren, welche heterogene Rhabdomyome, beziehungsweise Rhabdomyosarcome beschrieben, führen die Entstehung der neuen Muskelfasern auf verlagerte embryonale Muskelkeime zurück. Zur Begründung dieser Ansicht wird meistentheils Folgendes angeführt: 1) dass diese Tumoren meistentheils angeboren sind, oder in früherLebenszeit vorkommen, 2) dass sie in der Mehrzahl der Fälle an solchen Stellen gefunden werden, wo infolge einer Verschmelzung zweier Añagen für den Einschluss von fremdartigen Keimen die Gelegenheit günstig ist, 3 ) dass in derartigen Geschwülsten. verschiedene Gewebe neben einander vorkommen, und dieselben daher Mischgeschwülste genannt werden dürfen, 4) dass die mit Quersteifung versehenen Fasern und Zellen' in allen wichtiger Punkten mit den embryonalen Muskelfasern , übereinstimmen: (Cohnheim, Ribbert in seiner letzten Arbeit, Han a wolfensberger u. s. w.). Diese Auffassung passt für manche Rhabdomyosarcome ganz gut, und ich zweifle nicht, dass solcheTumoren wirklich vorkommen. Eine zweite Möglichkeit käme aber in Betracht, dass solche Rhabdomyome als homologe Bildungen aus einer embryonalen Anlage entstehen, wie es v. Recklinghausen zuerst an einer Herzgeschwulst nachgewiesen. hat; noch in neverer Zeit hat Kolisko bei einer Geschwolst im Myocard dieselbe Beschaffenheit der Fasern, wie an dem Herzfleisch eines 4 Wochen alten Foetus constatiren können.

Als ein drittes wäre die directe Neubildung vom präformirten Muskelgewebe aus zu berücksichtigen, wie es Bubl von seinen

1) Yirchow, ebendaselbst. 
beiden im willkürlichen Muskel entwickelten Myomen zugelassen hat. Die vierte Möglichkeit wäre eine Metaplasie von Zellen, und zwar der glatten Muskelzellen, oder eine Metaplasie der spindelförmigen Sarcomzellen zu quergestreiften Faserzellen. Wie verhält sich nun mein. Fall diesen Möglichkeiten gegenüber?

Zunächst kann man die dritte Möglichkeit der directen Neubildung aus dem präformirten Muskelgewebe ausschliessen. Die quergestreiften Faserzellen in meiner Geschwulst besitzen in keiner einzigen Beziehung irgend einen Zusammenhang mit den präformirten Muskelfasern. Ich habe ferner meine besondere Aufmerksamkeit darauf gelenkt, ob irgend eine Beziehung zwischen den Spindelzellen und präformirten Muskelfasern existirt, und ob die quergestreiften Geschwulstzellen indirecte Abkömmlinge von den präformirten Muskelfasern sein könnten. Die Umwandelung der Muskelfaser in Sarcomzellen, wie sie von Weber ${ }^{1}$ ) und Sakolow ${ }^{2}$ ) u. s. w. behauptet wird, ist wenigstens in diesem. Präparate trotz aller Mühe nicht zu constatiren gewesen. Denn die Muskelfasern erschienen im Gegensatz zu den wuchernden Zellenmassen einfach atrophisch, und ihre spindelartigen Zellen lagerten immer nur im interstitiellen Bindegewebe. Zweitens muss die Möglichkeit einer Metaplasie glatter Muskelfașern in quergestreifte ausgeschlossen werden. Ja, unter den spindeligen Sarcomzellen fand ich solche Zellen, welche den glatten Muskelzellen äusserst ähnlich erschienen, und die sich mit den quergestreiften Geschwulstzellen hätten identificiren lassen, wenn ihnen nur eine Querstreifung eigen gewesen wäre. Im "Rbobdomyosarcom" sind auch von früheren Autoren glatten Muskelfasern höchst ähnliche Zellen beschrieben worden (Marwhand, Langhans, Boström, Ribbert. Da auch ihre Anordnung öfters an den Aufbau eines Uterusmyoms erinnerte, namentlich in der Art der Durchkreuzung der Muskelfaserbündel, so führte Ribbert in seiner ersten Arbeit die

1) C. 0 . Weber: Ueber die Betheiligung der Muskelkörperchen und der quergestreiften Muskeln an den Neubildungen u. s. w. Dieses Archiv. Bd. XXXIX. S. 254 .

2) Sakolow: Ueber die Entwickelung des Sareoms in den Muskeln. Dieses Archiv, Bd. LVII, S. 321. 
quergestreiften Zellen und Fasern auf eine Metaplasie glatter Muskelzellen zurück, und Rindfleisch ${ }^{1}$ ) hat beobachtet, dass Uebergänge zwischen glatten und quergestreiften Muskelfasern vorkommen. Ich schliesse diese Möglichkeit nicht absolut aus, obwohl die quergestreiften Muskelzellen der Embryonalzeit keinen Zusammenhang mit den glatten Muskelzellen haben. Ist doch die Wucherung von Geschwulstzellen, wie die Regeneration eines Gewebes, eine ganz andere Sache, als die embryonale Gewebsentwicklung. Dennoch halte ich in meinem Falle diese Hypothese für höchst unwahrscheinlich, deswegen, weil die betroffenen Organe mit glattem Muskelzellgewebe nicht zu thun haben.

Somit stehen nur die beiden anderen Möglichkeiten zur Discussion, nehmlich die Entwickelung der quergestreiften Faserzellen aus embryonalen Muskelkeimen, und ihre metaplastische Entstehung aus den spindelförmigen Sarkomzellen. Zunächst ist nun, wie schon oben erwähnt, der allmähliche Uebergang von den typischen spindelförmigen Sarcomzellen in die quergestreiften Rhabdomyoma-Zellen sehr deutlich nachzuweisen, so dass man nicht berechtigt ist, jene Spindelzellen als eine besondere Species gegenüber den Rhabdomyom-Zellen anzusehen, oder anzunehmen, dass eine besondere embryonale Zellenart, die zur Ausbildung von Querstreifen befähigt, mit den gewöhnlichen Sarcomzellen vermengt wäre. Offenbar entstehen also die Rhabdomyom-Zellen auf metaplastischem Wege aus den spindelförmigen Sarcomzellen. Diese Annahme einer solchen Metaplasie von Zellen erscheint keineswegs als etwas Neues. Ausser der Angabe Virchow's, wovon oben die Rede war, wird im Perl'schen Lehrbuch ein "Uebergang von Spindelzellen in junge quergestreifte Muskelfasern " ${ }^{2}$ ) in Betracht gezogen; Birch-Hirschfeld bemerkt gleichfalls: „Uebergänge zu quergestreiften Muskelfasern kommen vor in Form von Spindeln mit quergestreiftem Protoplasma“".3) Aus diesen kurzen Notizen kann ich aber nicht sagen, ob die Autoren damit ausdrücken

1) Rindfleisch, Lehrbuch der path. Gewebelehre. 6. Auflage, 1886, S. 160.

2) Perl-Neelsen: Lehrbuch der allgemeinen Patbologie. 1886. S. 289.

3) Birch-Hirschfeld: Lebrbuch der allgemeinen patbolog. Anatomie. Leiprig, 1896, S. 243 . 
wollen, dass sich gewöhnliche Sarcomzellen in Muskelzellen, bezw. in die mit Querstreifung versehenen Zellen umwandeln können, oder ob sie ein solches Sarcomgewebe im Auge haben, welches aus jungen Muskelzellen entstanden sei.

Nachdem sich nun durch meine Untersuchung die Metaplasie der Rhabdomyomzellen aus den spindelförmigen Sarcomzellen als wichtigstes Resultat ergeben hatte, entstand die weitere Frage, woher diese Sarcome stammen, und damit auch die schwierige Frage nach der Pathogenese der Geschwulst überhaupt. Wir kommen dadurch auf die oben zuerst angeführte Annahme einer embryonalen Keim-Anlage zurück. Wenn man, wie es allgemein geschieht, den Satz zulässt, dass alle deutlich quergestreiften Fasern Muskelfasern sind, und dass die Muskelfasern immer aus specifischen Muskelzellen, entstanden sein müssen, kann man eigentlich die sämıntlichen Geschwulstzellen, ob quergestreift. oder nicht, als junge embryonale Muskelzellen betrachten, welche sich in verschiedenen Entwickelungs-Stadien befinden. Es ist ja höchst unwahrscheinlich, dass die Muskelzellen aus gewöhnlichen Bindegewebszellen angebildet werden können. Die Ansicht Zenkers's, dass für die regenerative Neubildung das interfasciculäre Bindegewebe die Matrix abgiebt, dass also Muskelzellen aus Bindegewebszellen entstehen können, ist durch spätere genauere Untersuchungen (Neumann, Fraisse, Leven, Nauwerck, Volkmann u. s. w.) hinfällig geworden. Die Anschauung, dass die Spindelzellensarcome nichts anders, als junge Myome oder Neurome wären, wurde ein Mal durch Billroth ${ }^{1}$ ) vertreten. Andere Autoren, wie Cornil und Ranvier ${ }^{2}$ ), bezeichnen das Sarcom als eine Geschwulst, die aus rein embryonalem Gewebe oder aus demjenigen der ersten Uebergänge zum fertigen Gewebs-Elemente besteht. Wolfensberger ${ }^{3}$ ) fasst ebenso alle Spindelzellen, die neben den quergestreiften Geschwulstzellen auftreten, als eine niedere Stufe embryonaler Muskelzellen auf. Schliesst man sich dieser Ansicht betreffs

1) Billroth: Die allgemeine chirurgische Pathologie u. Therapie. 1875. S. 711. S. auch Billroth, Dieses Archiv. IX. S. 178.

2) Cornil et Ranvier: Manuel d'histologie pathologique, 1881. I. p. 144.

3) Wolfensberger a. a. 0 . 
des Spindelzellensarcoms an, so kann man meinen Fall dahin erkären, dass oin gewisser Theil der embryonalen Muskelanlage nicht für die Gewebs-Entwickelung verbraucht wurae, und erst später durch eine Gelegenheitsursache, (z. B. durch den Reiz des Geschwürs und die Narbenbildung), in Wucherung gerathen ist, wobei gewisse Zellen durch weitere Differencirung (Querstreifung) ihre eigentliche Natur documentirten. Somit liegt in meinem Falle nicht eine Keim-Verirrung, wie man sonst für die meisten Rhabdomyome annimmt, sondern ein Differencirungs-Fehler vor. Die Endothel-Wucherung wäre erst durch den Reiz der Sarcombildung hervorgerufen worden. Obwohl die verschiedenen Momente, die man als Stütze für die Ansicht von der embryonalen Natur des Rhabdomyoms herangezogen hat, nicht alle in meinem Fall zutreffen, das hohe Alter, das deuthohe Vorhandensein eines veranlassenden Momentes und die Localisation, so kann daraus nicht ein Einwurf gegen die Richtigkeit meiner Erklärung des Geschehens abgeleitet werden.

Zum Schluss bezeichne ich in aller Kürze als die wichtigsten Ergebnisse meiner Beobachtung:

1. die Combination eines sogenanten Cylindroms und eines Rhabdomyosarcoms in der willkürlichen Körper-Musculatur eines alten Individuums, und das gleichzeitige Auftreten endotheliomatöser Anordnung;

2. die hyaline Degeneration (Cylindrombildung) verschiedeat Geschwulst-Elemente, sowohl der Blutgefässwand, als dec Bindegewebsfasern und Geschwulstzellen, besonders der spindeligen Zellen, vielleicht auch vereinzelter Muskelfaser-Reste;

3. die metaplastische Entstehung mit Querstreifung versehener Zellen und Fasern, welche morphologisch als junge Muskelfasern zu bezeichnen sind, aus spindelförmigen Sarcomzellen.

Ich spreche meinem hochverehrten Lehrer Herrn Prof. von Recklinghausen meinen herzlichsten Dank aus für die freundliche Ueberlassung des Materials, sowie für die liebenswürdige Unterstützung bei der Durchsicht der Präparate und bei der Anfertigung dieser Arbeit. 
Erklärung der Abbildungen auf Tafel XII:

Fig. 3. Woblausgebildete quergestreifte Fasern. Sie sind in zienolich reichlieber Menge mitten im BündeI der typiseben spindeligen Sarcomzellen nachgewiesen worden. Färnatoxylin-Eosin, Glycerin. Zeiss 4. D D.

Fig. 4. Verschieden gestaltete Spindelzellen and Fasem mit Querstreifung. Zupfpräparat. Härnatoxylin-Eosin, Glycerin. Zeiss 4. D D.

\section{Berichtigung:}

In meiner Arbeit „Ueber die Beziehungen der Myocarditis zu den Erkrankungen der Arterienwandungen" dieses Archivs, Band 159, Eeft 8.

S. 451 , Zeile 17 ist zu setzen:

"in vier von seinen Fällen" statt "in seinen 4 Fällen".

S. 488 , Zeile 7 von unten:

"Oestreich" statt "Oesterreich".

\section{XII.}

\section{Der intrapleurale Druck beim lebenden, gesunden Menschen.}

(Aus dem Krankenhause der jüdischen Gemeinde in Berlin. Dirigirender Arzt: Sanitãtsrath Dr. Lazarus.)

Von

Dr. E. Aron, Assistenzarzt.

Vor einer Reihe von Jahren fand $\mathrm{ich}^{1}$ ) bei einer Patientin, welche wegen eines Empyems der Pleurahöhle wittelst der Bülau'schen Heberdrainage behandelt wurde, als Mittelzahlen für den intrapleuralen Druck am lebenden Menschen:

auf der Höhe der Inspiration - 4,0 der Exspiration - 1,9 $\mathrm{mm} \mathrm{Hg}$ im Liegen;

1) Dieses Archiv Bd. 126. 1891. p. 517. Ueber einen Versuch, den intrapleuralen Druck am lebenden Menschen za messen. 